\title{
De saltos paradigmáticos a redes de cuidado: un extraño recorrido
}

\section{From paradigmatic leaps to care networks: a strange journey}

\author{
Leticia O. Minhot ${ }^{1}$ \\ Universidad Nacional de Córdoba \\ (Facultad de Psicología/Facultad de Ciencias Sociales) \\ E-mail: leminhot@gmail.com
}

\begin{abstract}
Resumo
Este trabalho tem como objetivo mostrar quão forte é a influência do pensamento de Loparic em minhas reflexões e quantos dos problemas que trabalho são atravessados por sua filosofia. É impossível falar sobre os caminhos das idéias percorridos sem falar da forte presença de Zeljko Loparic neles. Meu objetivo aqui é mostrar como passei de agradáveis exercícios epistemológicos para ocupações éticas desconfortáveis. Começarei descrevendo os primeiros e depois analisando as preocupações que surgiram e que me levaram à ética do cuidado, indicando como as reflexões filosóficas de Loparic estavam acompanhando esses itinerários.
\end{abstract}

Palavras chaves

Paradigmas, Kuhn, ética nos cuidados, comunidade solidária

Resumen

Este trabajo tiene por objetivo mostrar cuan fuerte es la influencia del pensamiento de Loparic en mis reflexiones y cómo muchas de las problemáticas que trabajo están atravesadas por su filosofía. Es imposible hablar del camino de ideas andado sin hablar de la fuerte presencia de Zeljko Loparic en estos trayectos. Mi propósito aquí es mostrar cómo pasé de placenteros ejercicios epistemológicas a incómodas ocupaciones éticas. Comenzaré describiendo los primeros para luego analizar las inquietudes que fueron surgiendo y que me llevaron a la ética del cuidado, indicando cómo las reflexiones filosóficas de Loparic fueron acompañando estos itinerarios.

Palabras claves

\footnotetext{
${ }^{1}$ Licenciada en Filosofía. Facultad de Filosofía y Humanidades, Universidad Nacional de Córdoba, 1985. Doctora en Filosofia. Universidade Estadual de Campinas, Brasil, 2001, con la tesis "Uma reconstrução das teorias psicanalíticas de Freud segundo as categorías da matriz disciplinar de Kuhn". Dirigida por Zejko Loparic. Cargos actuales:Profesora Titular da Cátedra de Problemas Epistemológicos de la Psicología. Facultad de Psicología. Universidad Nacional de Córdoba.Profesora Titular: Cátedra de Concepciones Filosóficas. Escuela de Trabajo Social. Facultad de Ciencias Sociales. Universidad Nacional de Córdoba.Investigación:2018-2021: Proyecto de Investigación: Transgresión como emancipación: resistencia creativa ante el capitalismo como cuestión ética. Directora. Subsidio SECYTUNC.2016-2017: Proyecto de investigación: Transgresión y cuidado: la comunidad solidaria. Directora. Subsidio SECYT-UNC2014-2015: Programa de investigación: La ética del cuidado: sus fundamentos y sus implicancias en contextos de vulnerabilidad social. Directora. Subsidio SECyT UNC 2014-2015: Proyecto de investigación: Transgresión y cuidado: la ética del cuidado y la creatividad en la reformulación de la transgresión. Directora. Subsidio SECyT UNC
} 
Paradigmas, Kuhn, ética del cuidado, comunidad solidaria

\section{Introducción}

Zeljko Loparic es mi orientador de doctorado en la Universidade Estadual de Campinas, Brasil, entre los años 1997 y 2001a. Mi tesis consiste en una reconstrucción kuhniana de las teorías clínicas de S. Freud. Tal investigación culmina con mi doctorado en Filosofía y con un texto, (Minhot, 2003), que contiene lo fundamental allí trabajado. Los encuentros con mi orientador siempre se han caracterizado por ser enriquecedores y por aportar a mi crecimiento personal e intelectual. Por aquella época yo ya había trabajado aspectos formales de la reconstrucción de teorías y, de hecho, continúo luego de mi doctorado, siguiendo la línea del estructuralismo metateórico de Moulines, Balzer, Lorenzano P., y otros. La dirección de Loparic me lleva a focalizar en lo que es, quizás, el auténtico elemento revolucionario de la concepción kuhniana de la ciencia: esto es, los ejemplares. Ver la ciencia como una actividad de resolución de problemas es la noción articuladora de mi trabajo por aquel momento. A pesar de que algunos trabajos de Kuhn plantean que tal resolución de problemas no estarían presentes en el psicoanálisis y, a pesar de que algunos filósofos plantean que la estructura de la matriz disciplinar no es aplicable al psicoanálisis, sin embargo, ni muchos autores del estructuralismo metateórico, ni Loparic, ni yo estuvimos de acuerdo con esa idea. Y mi tesis, más que debatir la posibilidad de tal aplicación, consiste en la tarea de reconstruir, con dicha estructura kuhniana, las teorías freudianas. En el próximo apartado muestro las consecuencias de focalizar en los ejemplares más que en las teorías mismas.

Winnicott es un autor que Loparic siempre me estimula para trabajar pero, en la época en que me encuentro haciendo mi doctorado, no lo considero y decido centrarme en Freud, tal como lo planificamos originalmente. Es a mi regreso a Argentina cuando comienzo a leer con interés a Winnicott. Y me dedico al trabajo de comparar las matrices disicplinarias freudiana y winnicottiana siguiendo la heurística de Loparic, según la cual, Winnicott produce una revolución kuhniana en el psicoanálisis. En el apartado 3 expongo algunos resultados de esta línea de trabajo.

Profundizar en el pensamiento de Winnicott y trabajar en la Teoría de la Maduración que este autor elabora despierta en mí un nuevo interés. El cuidado 
comienza a aparecer en mi horizonte filosófico de un modo persistente. La ética del cuidado significa un punto de quiebre con mi línea de trabajo anterior. Nuevos problemas filosóficos surgen y comienzan a acicatearme para recorridos que nunca hubiera pensado. En el apartado 4 cuento de qué modo es que llego, gracias a Loparic, a la ética del cuidado. Hoy continúo con esta temática, aunque ya no tan focalizada en el psicoanálisis y abordando otros problemas. Claramente, el pensamiento de Loparic ha marcado mi derrotero filosófico, por supuesto es inocente de todas mis equivocaciones y malas comprensiones, pero si he llegado a algún lugar ha sido gracias a su filosofía y a su generosidad.

2. Los ejemplares en psicoanálisis

Como señalo en la introducción, considerar a la ciencia como una tarea de resolución de problemas es una de las nociones más decisivas en mi viaje filosófico. Es el concepto desde el cual articulo mi trabajo de doctorado. Si centramos en los ejemplares podemos encontrar un Kuhn que ha tenido un alto impacto en la filosofía. Irzik y Grünberg (1995) si bien establecen las cercanías entre Kuhn y Carnap -cercanías más con el Kuhn posterior al de las Revoluciones- señalan que, justamente, la idea kuhniana novedosa consiste en identificar las habilidades adquiridas para ver relaciones de semejanza y a los ejemplares como elemento central de la práctica científica. Acuerdan en que no hay nada en la concepción carnapiana de teoría o de estructura lingüística que se corresponda con los ejemplares, por lo tanto, Kuhn está enteramente justificado cuando pretende la originalidad de sus nociones (1995, p. 298). Para Rouse (1987, pp.38-39) una lectura de Kuhn desde los ejemplares permite una comprensión de la ciencia totalmente diferente a la centrada en las construcciones teóricas que ha predominado en la tradición epistemológica dominante. Rouse enfatiza que las matrices disciplinares son el conjunto de los modos ejemplares de conceptuar e intervenir en contextos empíricos particulares que los miembros de una comunidad han consensuado. A su vez, Irzik y Grünberg destacan que mientras Carnap apela a reglas de correspondencia para otorgar significado empírico a los términos teóricos, Kuhn considera que éste no es el único modo, ni el más eficaz, por el que las teorías formalizadas adquieren contenido empírico. El modo de intervención en un dominio empírico determinado no reside, principalmente, en las reglas: los ejemplares proveen un modo mucho más adecuado para captar cómo una teoría aplica a la 
naturaleza. (Kuhn, 1977/1982, pp.290 y ss) Rouse señala que en los ejemplos paradigmáticos los acuerdos no son respecto a compromisos teóricos sino, más bien, a modos de intervención. Aceptar una matriz disciplinar consiste en adquirir y aplicar estas habilidades más que entender y creer un enunciado. Para este Kuhn, la ciencia normal envuelve prácticas compartidas, no creencias compartidas, por eso, Rouse se compromete con la idea que sostiene que el cambio de un paradigma a otro no es una conversión a nuevas creencias sino una metamorfosis de una forma de vida. A su vez, Loparic (2001a) avanza en el esbozo de la matriz disciplinar de Winnicott y su énfasis es puesto en la idea kuhniana de la ciencia normal como una actividad dedicada a la resolución de problemas. Loparic enfatiza la idea de cómo un problema se torna científico cuando es un rompecabezas cuya solución es legitimada por un marco teórico determinado, los científicos se entrenan para encontrar esa solución. La solución científica a esos rompecabezas se encuentra en los ejemplares los cuales conllevan un conocimiento tácito que se trasmite en el entrenamiento de los científicos y que describe las actividades cotidianas de los mismos en su campo disciplinar. Loparic, en el trabajo mencionado y en otros sigue el criterio de cientificidad kuhniano -esto es, una práctica es científica cuando, siguiendo soluciones previas, resuelve problemas- para probar que en el psicoanálisis hay tales rompecabezas. Así, el eje de su lectura de Kuhn sigue esta tradición que enfatiza las prácticas basadas en ejemplares y se aparta de la corriente epistemológica que ha entendido paradigma como concepción o visión de mundo.

En mi tesis se considera que son los casos que presenta Freud los ejemplares de su teoría. Se dividen en dos grupos: los que datan del período 1889-1892 y los que presenta en el período 1905-1918 y se muestra que el problema-solución es diferente en ambos grupos. De estos casos paradigmáticos emerge la estructura de datos que la comunidad luego acepta. Si la adopción de la matriz disciplinar implica que la base consensual por la que existe una comunidad sólo se da cuando todos ven lo mismo podemos concluir que cuando, ante un conjunto de determinados síntomas, un grupo ve alguna versión del complejo paterno presente en fantasías como en el segundo grupo de ejemplares- y otro ve secuelas de una vivencia traumática -como el primer grupo de ejemplares- no están viendo lo mismo y trabajan con estructuras de datos diferentes, por lo tanto, son comunidades inconmensurables. El conjunto de síntomas está organizado desde diferentes esquemas de ley, la percepción organiza de modo diferente lo que se ve. El conjunto 
de ejemplares determinado por la teoría del trauma representa un conjunto de soluciones exitosas distinto del que representa el conjunto de ejemplares para la teoría del complejo paterno. Las redes de semejanzas en las cuáles son educados los investigadores futuros son diferentes conforme se parta de un grupo u otro de ejemplares. Así, los terapeutas entrenados en un conjunto o en otro de ejemplares, ven, delante del mismo paciente, pacientes diferentes y sus prácticas, en consecuencia, también son diferentes. Esta conclusión a la que llego en mi investigación produce largos debates con Loparic quien ya, por esa época trabaja en la tesis de que en el psicoanálisis sólo hay dos paradigmas. En rigor, mi conclusión no es tan opuesta a la premisa de Loparic. En primer lugar, para Freud, el psicoanálisis nace propiamente cuando abandona la teoría de la seducción, por lo que el psicoanálisis freudiano se caracteriza propiamente por la matriz disciplinar que contiene como ejemplares los casos de Dora, el Hombre de los lobos, el Hombre de las Ratas, Juancito, el caso Schreber. En consecuencia, la primera matriz disciplinar freudiana no es psicoanalista propiamente por lo que mi conclusión no impide trabajar bajo la heurística de Loparic de dos matrices disciplinares psicoanalíticas: la de Freud y la de Winnicott. En segundo lugar, cuando profundizamos en las prácticas cotidianas podemos percibir que lo fundamental es cómo resuelven los problemas los científicos, esto no complica la idea según la cual, en el plano de las creencias, el paradigma no es tan liso y homogéneo. Mi investigación me lleva a ver muchos de los pliegues presentes en la obra freudiana aunque sean sólo dos los paradigmas de resolución de problemas: el de la seducción o trauma y el psicoanalítico.

\section{Comparando paradigmas}

Como ya mencioné, es recién a mi regreso a Argentina cuando comienzo a dedicarme al estudio de la Teoría de la Maduración de Winnicott. Me dedico al trabajo de comparar las matrices disciplinares de Freud y de Winnicott bajo la heurística de Loparic (1995, 1996, 1997, 1998, 1999, 2000, 2001b, 2002, 2003b, 2005b, 2006b, 2008, entre otros) que defiende la presencia de una revolución paradigmática en el psicoanálisis producida por la Teoría de la Maduración. Me centro en el estudio de algunos de los elementos de la matriz winnicottiana, fundamentalmente los modelos ontológicos, pero también de las comunidades fundadas por los ejemplares de Winnicott. Como señala Loparic (2002), la relación 
madre-bebé abre la posibilidad para que problemas que el psicoanálisis tradicional no podía considerar como tales puedan ahora emerger: ¿qué es lo que determina el sentimiento de sentirse vivo o real?

En El Psicoanálisis, las ontologías de la ciencia y el pensamiento de la existencia (Minhot, 2011) la comparación se centra en las estructuras ontológicas filosóficas que subyacen a sus modelos ontológicos más que en estos mismos. Convencida de la importancia para el psicoanálisis de un diálogo entre los modelos ontológicos disciplinares y una ontología filosófica referida a la existencia humana si bien tomo otros autores, la posibilidad del diálogo entre el psicoanálisis y la filosofía es desarrollada por Loparic (2006a entre otros)- busco comparar la idea de ser humane que subyace a los posicionamientos teóricos psicoanalíticos. El pretexto de este cruce es la preocupación por el pathos del ser humano que ambos discursos comparten. Desde esta intersección se compara el modelo individuo-deseo de Freud -que lleva al mundo psíquico el determinismo que Kant le atribuye solamente al mundo natural- con el ambiente que permite la continuidad del ser. En el modelo freudiano hay un determinismo entre representaciones que, encadenadas unas a otras, permiten la etiología psíquica y son el fundamento de la interpretación psicoanalítica. El significado consiste en estar determinado causalmente, en estar en ese encadenamiento. La interpretación radica en encontrar las representaciones ocultas de la propia vida anímica. La ontología pulsional es una ontología internalista que se basa en una ontología de substancia individual: la sexualidad es un contenido fundamental del aparato psíquico del individuo entendido ontogenéticamente. En oposición, el psicoanálisis winicottiano no presenta una metapsicología, no presenta un modelo a priori de la psiquis y no se inscribe en la oposición interno-externo. El concepto ambiente, clave de la Teoría de la Maduración, es lo que permite o no que se llegue a ser un individuo, por lo cual éste es un logro que puede darse o no. El interrogante ¿Qué sentido tiene la vida y qué hace que sea digna de ser vivida?, como destaca Olivera Días (2003, p. 96), es propio de todos los seres humanos y, son justamente los psicóticos, más que nadie, con sus dudas permanentes entre el vivir y el no vivir, los que nos enfrentan a esta cuestión. Lejos de una concepción determinista, la pregunta por el sentido tiene como condición de posibilidad un cuerpo vivo, no mecánico. Así, mientras el psicoanálisis freudiano elabora los sentidos por medio de una causalidad en la vida 
anímica, el psicoanálisis winnicottiano lo hace por medio del vínculo que hizo posible el cuerpo habitado, único dador de sentidos.

Ontología relacional en la formulación sobre el juego de D. Winnicott (Minhot, 2018) se centra en la estructura ontológica de la Teoría de la Maduración, la cual es caracterizada como relacional. Si bien Loparic (2013) hace énfasis en la relacionalidad del cuidado, en este trabajo lo relacional es abordado en el sentido epistemológico y no metafísico, por eso se habla de modelos ontológicos. Un rasgo de estos modelos consiste en que no parten de entidades separables consideradas como dadas sino que, más bien, presentan procesos por los cuales se llega a ser. Estos procesos no tienen lugar a partir de un núcleo inicial, en ese sentido, no pueden considerarse como que modelizan ontogénesis. Esto puede verse claramente en la estructura del concepto ambiente, base de la confianza y, por ende, de la creatividad que se manifiesta desde el comienzo en el jugar.

Otro de los aspectos que abordo en mi investigación sobre esta matriz disciplinar es el de las comunidades psicoanalíticas winnicottinas. Cambiar el eje desde el Complejo de Edipo a la dependencia del bebé genera un nuevo lenguaje para hablar del ser humane en general y de la enfermedad psíquica que puede afectarlo. El cambio de matriz implica el cambio de un lenguaje que habla en términos de deseo a uno que habla en términos de necesidad. De uno que habla en términos de pulsiones a uno que habla en términos de ambiente. Este cambio da lugar a la emergencia de una nueva comunidad cuyos adherentes se comunican en ese nuevo lenguaje y resuelven sus problemas en base a los ejemplares winnicottianos. La idea central de la interpretación que de Winnicott hace la comunidad de São Paulo sostiene que el autor desarrolla su propia teoría -la Teoría de la Maduración- la cual le da al psicoanálisis unidad y articulación. (Dias, Loparic, 2011: 15) Esto implica que el campo disciplinar se estructura de un modo diferente al tradicional. Aparecen nuevas circulaciones mediante las cuales se van forjando nuevos consensos y se trasmiten nuevas formas de reconocimiento y resolución de problemas. El Centro Winnicott de São Paulo, la Escola Winnicottiana de Psicanálisis, la Sociedade Brasileira de Psicanálisis Winnicottiana, la SWW editorial, la revista Natureza Humana son algunas de las circulaciones por medio de las cuales la comunidad sella el consenso que está contenido en la matriz disciplinaria winnicottiana. Así, hablar de paradigma winnicottiano no refiere solamente a la Teoría de la Maduración sino a todas estas circulaciones. Pues no hay 
matriz sin sociedad pues la matriz es lo que lo compartido y el modo en que se resuelven los problemas -los ejemplares- es lo que fundamenta y da identidad a esa comunidad. Esto está presente tanto en Minhot (2018) como en Over the shoulders of a gigant (Minhot, 2017). En Ontología relacional en la formulación sobre el juego de D. Winnicott, en debate con Green -para quien hay ausencia de una verdadera escuela winnicottiana- se le reprocha que se centra solamente en la British Society y deja de lado lo que sucede en otras partes del mundo, por eso es que no puede registrar la presencia de verdadera escuela winnicottiana, entendiendo por esto comunidades organizadas por la matriz disciplinar derivada de la Teoría de la Maduración. Quizás, conservar un cuerpo psicoanalítico unido, aunque sea en apariencia, es un objetivo político de la British Society pero que dicho objetivo no provoque negligencia de circulaciones de las ideas de Winnicott que han cristalizado en auténticas comunidades psicoanalíticas. Comunidades que hicieron de los casos de Winnicott verdaderos ejemplares seminales. En este sentido kuhniano, comunidad científica admite en su seno diferencias pero se definen por lo que comparten, esto es, la matriz disciplinar y, con ella, modos específicos de resolución de problemas. En Over the shoulders of a gigant, como señalamos, se reconoce que la Teoría de la Maduración sola no habría alcanzado para establecer una práctica científica normal si no guiara la investigación psicoanalítica de una comunidad. Desde este lugar se aborda el futuro de la matriz disciplinar del psicoanálisis winnicottiano. Focalizando cómo, desde Winnicott, podemos ir más allá de Winnicott, fundamentalmente buscando nuevas áreas de aplicación de la Teoría de la Maduración y la capacidad de ésta para resolver nuevos problemas, fundamentalmente problemas que aquejan a las sociedades actuales. Hoy, releyendo estos resultados no queda más que destacar no sólo el aporte teórico de Loparic como también el haber sido un actor fundamental en la gestación de la comunidad winnicottiana internacional.

\section{El cuidado}

El trabajo de comparación de matrices disciplinares y profundización en la matriz generada por la Teoría de la Maduración me va llevando a enfocar en el tema del cuidado. Esta noción comienza a ejercer una gravitación en mi investigación. La ética del cuidado provoca una ruptura con respecto a mis preocupaciones epistemológicas. Se abren paso interrogantes que antes no son considerados. 
Reflexionar sobre la ética del cuidado no es una senda que se transite sin una transformación personal, por eso, que Loparic (1989, 1995a, 2003a, 2005a, 2007, 2013), mediante sus reflexiones filosóficas, haya abierto tales posibilidades es algo con lo cual siempre estaré agradecida.

La preocupación ontológica guía la investigación que resulta en el trabajo Dos concepciones ontológicas de sociedad (Minhot, 2014). Se compara la estructura ontológica de la sociedad en la teoría freudiana y la que se deriva de la teoría de la maduración. Freud, siguiendo el relato paradigmático de la modernidad parte del individuo. Sólo que aquí la comparación no nos lleva a la vereda opuesta esperada. Ciertas consideraciones de Winnicott respecto de la sociedad nos llevan a sospechar que su modo de concebirla está en contradicción con la estructura ontológica relacional que prevalece en la Teoría de la Maduración. La comparación entre matrices disciplinares se continúa en las reflexiones sobre las éticas de la justicia y las éticas del cuidado. En Ética y psicoanálisis: supuestos filosóficos compartidos (Minhot, 2013) se muestra un parentesco filosófico entre, por un lado, las teorías freudianas con las éticas de la justicia y, por otro lado, la Teoría de la Maduración y las éticas del cuidado. El primer vínculo se funda en tres premisas: sujeto humano identificado con individuo, universalización y formalización tanto del sujeto como de normas que resulta en la abstracción de los mismos y un sujeto concebido como agresor. El segundo parentesco se basa en los siguientes enunciados: el cuidado es una actividad concreta y no un modelo abstracto; el sujeto está en un contexto y las necesidades concretas constituyen el rasgo principal del sujeto como ser en relación. Profundizar en estas premisas me permite comenzar a percibir la potencialidad del cuidado para la constitución de nuevas subjetividades, una inquietud que, en investigaciones posteriores, me lleva a confrontar los planteos éticos de Foucault.

Las investigaciones en temas relativos a la ética del cuidado y a la estructura ontológica de la sociedad me conducen a la preocupación de buscar un modo de concebir una comunidad solidaria y a enfocar en la Teoría de la Maduración para encontrar sus bases. En Dos concepciones ontológicas de sociedad comienzo a pensar en la comunidad solidaria como una red de cuidados. El trabajo de elucidar tanto comunidad -diferente de sociedad- como comunidad solidaria revela la importancia, en un mundo como el actual, de la promoción del cuidado como forma vincular de ser y esto comienza a transformarse en una demanda a nuestras reflexiones filosóficas. Si vivimos en una comunidad en la que el sostén entre unos 
y otros es su pilar fundamental entonces, es muy probable que, quienes sean sus miembros desarrollen condiciones para vivir creativamente frente a los embates que nos desafían hoy. La perspectiva en la cual hoy estoy más comprometida considera que el cuidado no supone que seamos seres racionales, ni siquiera que seamos humanos, no supone ninguna clasificación. No es una obligación, es un modo de ser en el que nos reconocemos como seres-con otros seres pues, como destaca Loparic (2013), su dimensión relacional es lo que nos ha constituido, aunque Loparic conserva un cierto humanismo heideggeriano del que yo me aparto. Así, construir la noción de cuidado como una acción necesaria para desarrollar las condiciones para promover una sociedad solidaria que no discrimine entre especies, es el eje de mis reflexiones actuales. Esta articulación me lleva a vislumbrar un proyecto político donde el cuidado no constituye un deber moral sino un modo de existencia. En este sentido, es muy importante llevar a Winnicott más allá de los límites de la clínica y es en lo que me enfoco en el presente.

Quiero terminar con dos citas provenientes de diferentes textos suyos que cada vez que las leo siento la fuerza inspiradora de un horizonte filosófico.

Fundamentar algo significa enraizá-lo, dar-lhe sustentação e proteção (2004, p. 27) ... o ser-com a-fim-deoutros é sempre, na origem, um movimento de abertura de possibilidades para o outro, um cuidar. (2004, p. 61) ... Na origem, o ter que ser no sentido de cuidar é um traço da "relacionalidade" que é inerente ao existir humano e que, como tal, prescinde de condicionamentos externos. (2013, p. 52)

Estoy en deuda con Loparic por haberme puesto en la senda filosófica del cuidado pues todas mis investigaciones actuales, de un modo u otro, están atravesadas por esta noción. El trabajo filosófico de Loparic ha señalado una dirección a mis interrogantes y reflexiones tanto en el pasado como ahora.

\section{Referencias}

Días, E. O. (2003). A teoria do amadurecimento de D. W. Winnicott. Rio de Janeiro: Imago. 
Dias, E. O. \& Loparic, Z. (orgs.) (2011). Prefácio. In E. O. Dias \& Z. Loparic (orgs.), Winnicott na Escola de São Paulo (pp. 9-17). São Paulo: DWW editorial.

Irzik, G \& Grünberg, T. (1995). Carnap and Kuhn: Arch Enemies or Close Allies? The British Journal for the Philosophy of Science, 46(3), 285-307. Disponível em: http://www.jstor.org/stable/687658. Acessado em: 26/09/2019.

Kuhn, T. (1977). The Essential Tension. Selected Studies in Scientific Tradition and Change. Chicago: University of Chicago Press. (Edición en español: Kuhn, T. (1982). La tension esencial: estudios selectos sobre la tradición y el cambio en el ámbito de la ciencia. México: Fondo de Cultura Económica.)

Loparic, Z. (1989). Lacan e a ética do desejo perverso. In F. Hisgail (org.), 14 conferências sobre Jaques Lacan (pp. 29-52). São Paulo: Escuta.

Loparic, Z. (1995a). Ética e finitude. São Paulo: EDUC.

Loparic, Z. (1995b). Winnicott e o pensamento pós-metafísico. Psicologia USP, 6(2), $36-61$.

Loparic, Z. (1996). Winnicott: uma psicanálise não edipiana. Percurso, 17, 41-47.

Loparic, Z. (1997). Winnicott e Melanie Klein: conflito de paradigmas. In I. E. M. Catafesta (org.), A clínica e a pesquisa no final do século: Winnicott e a universidade. São Paulo: Lemos.

Loparic, Z. (1998). Psicanálise: uma leitura heideggeriana. Veritas, 43, 25-41.

Loparic, Z. (1999). A teoria winnicottiana do amadurecimento pessoal. Revista infanto, 7(1), 21-23.

Loparic, Z. (2000). O “animal humano”. Natureza humana, 2 (2), 351-397.

Loparic, Z. (2001a). Esboço do paradigma winnicottiano. Cadernos de história e filosofia da ciência, 11(2) pp. 7-58.

Loparic, Z. (2001b). Theodor Lipps: uma fonte esquecida do paradigma freudiano. Natureza humana, 3(2), 315-331.

Loparic, Z. (2002). Winnicott's paradigm outlined. Revista Latinoamericana.

Psicopatología Fundamental, vol. 1, 61-98.

Loparic, Z. (2003a). Sobre a responsabilidade. Porto Alegre: EDIPUCRS.

Loparic, Z. (2003b). De Kant a Freud: um roteiro. Natureza humana, 5(1), 231-245.

Loparic, Z. (2004). Ética e finitude. São Paulo: Escuta.

Loparic, Z. (2005a) A fabricação dos humanos. Manuscrito, 28 (2), 339-365.

Loparic, Z. (2005b). Elementos da teoria winnicottiana da sexualidade. Natureza humana, 6(2), 311-358. 
Loparic, Z. (2006a) Heidegger e Winnicott. Winnicott e-prints, série 2, 2(1), 28-44.

Loparic, Z. (2006b). De Freud a Winnicott: aspectos de uma mudança paradigmática. Natureza humana, 8 (especial), 21-47.

Loparic, Z. (2007). Objetificação e intolerância. Natureza humana, 9(1), 51-95.

Loparic, Z. (2008). O paradigma winnicottiano e o futuro da psicanálise. Revista brasileira de psicanálise, 42 (1), 137-150.

Loparic, Z. (2013). A ética da lei e a ética do cuidado. In Z. Loparic (org.), Winnicott e a ética do cuidado (pp. 19-53). São Paulo: DWW editorial.

Minhot, L. (2003). La mirada psicoanalítica. Un análisis kuhniano del psicoanálisis de Freud. Córdoba: Brujas.

Minhot, L. (2011). El psicoanálisis, las ontologías de la ciencia y el pensamiento de la existencia. Natureza humana, 13(2).

Minhot, L. (2013). Ética y Psicoanálisis: supuestos filosóficos compartidos. In Z. Loparic (org.), Winnicott e a ética do cuidado (pp. 135-158). São Paulo: DWW editorial.

Minhot, L. (2014). Dos concepciones ontológicas de sociedad. Natureza humana, 16(1).

Minhot, L. (2017). Over the Shoulders of a Gigant. In Z. Loparic \& C. R. Vasconcelos (orgs.), Winnicott and the Future of Psychoanalysis. São Paulo: IWA Books.

Minhot, L. (2018). Ontología relacional en la formulación sobre el juego de D.

Winnicott. In C. R. Vasconcelos (org,), Ontologia e Psicanálise: diálogos possíveis (pp. 160-175). São Paulo: DWW editorial.

Rouse (1987). Knowledge and Power: Toward a Political Philosphy of Science. Ithaca: Cornell University Press. 